\title{
On Multicast Trees: Structure and Size Estimation
}

\author{
Danny Dolev Osnat Mokryn \\ School of Engineering and Comp. Sc. \\ Hebrew University \\ Jerusalem, Israel \\ \{dolev,osnaty\}@cs.huji.ac.il
}

\author{
Yuval Shavitt \\ Dept. of Electrical Eng. - Systems \\ Tel-Aviv University \\ Tel-Aviv, Israel \\ shavitt@eng.tau.ac.il
}

\begin{abstract}
This work presents a thorough investigation of the structure of multicast trees cut from the Internet and power-law topologies. Based on both generated topologies and real Internet data, we characterize the structure of such trees and show that they obey the rank-degree power law; that most high degree tree nodes are concentrated in a low diameter neighborhood; and that the sub-tree size also obeys a power law.

Our most surprising empirical finding suggests that there is a linear ratio between the number of highdegree network nodes, namely nodes whose tree degree is higher than some constant, and the number of leaf nodes in the multicast tree (clients). We also derive this ratio analytically. Based on this finding, we develop the Fast Algorithm, that estimates the number of clients, and show that it converges faster than one round trip delay from the root to a randomly selected client.
\end{abstract}

\section{INTRODUCTION}

There are several inhibitors to the commercial use of multicast protocols. While it is clear that multicast is beneficial for transmitting the same information to large groups, its exact gain over unicast has not yet been determined [1], [2], [3]. Network suppliers lack a fast and efficient way to estimate the size of large multicast groups, and the research community lacks reliable tree models.

We present here a thorough investigation we performed on the structure and characteristics of multicast trees cut from generated power law topologies and the Internet. While the exact nature of the Internet topology is in debate, our results show that the partial views we have from the Internet obey the power laws found by [4]. These results were also verified by [5], [6], [7], who conducted further investigations. Moreover, trees cut from the Internet and from the generated topologies had similar characteristics.

\footnotetext{
${ }^{0}$ This research was supported by the United States - Israel Binational Science Foundation (BSF), Israel; The Checkpoint PhD feelowship grant, Israel; The Intel COMM Grant - Internet Network/Transport Layer and QoS Environment (IXA).
}

We found that trees cut from such topologies and the Internet obey a degree-rank and sub-tree size-rank power law distributions ${ }^{1}$. We also found that the distance distribution of nodes from the root node resembles a Gamma distribution, as shown previously for the Internet [7]. We observed that nodes with degree higher than five tend to be rare in the resulting trees. These high degree nodes can always be found in several adjacent rings, which reside typically at the core of the network, and in the near vicinity of the tree root.

Our most intriguing result finds a linear ratio between the number of high degree nodes in the tree and the number of clients ${ }^{2}$. The result is shown to be valid for trees cut from scale-free topologies that were generated with various parameters, as well as for experiments conducted on the Internet itself. We further verify this ratio analytically for power law trees. Based on the tree topological characteristics we found, we suggest the Fast Algorithm for estimating the size of large multicast groups. We analyze the algorithm's expected delay in the Internet, which sums up to less than the round trip delay from the root node of the tree to a random client at the edge of the network.

Estimating the population size of large multicast trees can improve the performance of feedback mechanisms of protocols such as RTP [8] and SRM [9]. Current feedback supression solutions for RTCP use timers at the receivers [14], [15]. Our sender based estimation produces a much faster estimation that can be propagated to the receivers and eliminate the need for such timers. Often, feedback suppression protocols are based on similar techniques as polling based estimation algorithms [11], [12], [13] and thus can use our faster estimation instead. Fast estimation may also be beneficial to forward error correction protocols [10].

Our suggested estimation algorithm offers an alter-

\footnotetext{
${ }^{1}$ Note that rank-degree and frequency-degree power laws can be derived from each other [6].

${ }^{2}$ We note by clients the group of routers that directly attach clients.
} 
native approach by using the topological characteristics to obtain an estimation on the number of receivers (rather than a specific population count). It does not aggregate information at the router level, but rather polls the high degree routers in the multicast tree. Due to the characteristics of the tree, the furthest high degree router is closer to the root node than clients attaching routers that branch from it. The algorithm adapts itself to dynamic topological changes, and can therefore reflect changes in the session size, as does the population sampling algorithm suggested in [16].

To the best of our knowledge, this is the first time that the existence of a power law in the underlying topology is leveraged to construct an algorithm. We believe that more such algorithms can be developed in the future for a variety of purposes.

\section{EMPIRICAL CHARACTERISTICS OF MUltiCAST TREES}

This section details our findings on the structure of multicast trees cut from generated power law topologies, as well as the Internet. These findings are the basis for the estimation method we present in Section III, and are of interest in their own right.

Little work has been done on modeling and characterizing multicast trees. Chalmers and Almeroth [3] investigated the branching characteristics of Internet multicast trees on the MBone and their impact on multicast efficiency. They found that multicast trees tend to have low average internal degree that grows logarithmically with the number of receivers in the tree, and a maximum height of approximately 23 nodes. They also found a high frequency of "relay" nodes that have a degree of two throughout the tree. In previous work, Pansiot and Grad, who constructed trees from a graph based on true routing paths in the Internet, also showed a high frequency of relay nodes in the tree graphs [17].

\section{A. Topology and Tree Generation}

Our method for producing trees is the following. First, we generate power law topologies based on the Notre-Dame model [18]. The model specifies 4 parameters: $a_{0}, a, p$ and $q^{3}$. Where $a_{0}$ is the initial number of detached nodes, and $a$ is the initial connectivity of a node. When a link is added, one of its end points is chosen randomly, and the other with probability that is proportional to the nodes degree. This reflects the fact that new links often attach to popular (high degree) nodes. The growth model is the following: with probability $p, a$ new links are added to the topology. With probability $q, a$ links are rewired, and with probability $1-p-q$ a new node with $a$ links is added. Note that

\footnotetext{
${ }^{3}$ The notations in [18] are $m_{0}, m, p$ and $q$.
}

$a, p$ and $q$ determine the average degree of the nodes. We created a vast range of topologies, but concentrated on several parameter combinations that can be roughly described as very sparse (VS), Internet like sparse (IS) and less sparse (LS). Table I summarizes the main characteristics of the topologies used in this paper.

From these underlying topologies, we create the trees in the following manner. For each predetermined size of client population we choose a root node and a set of clients. Using Dijkstra's algorithm we build the shortest path tree from the root to the clients. To create a set of trees that realistically resemble Internet trees, we defined four basic tree types. These types are based on the rank of the root node and the clients nodes. The rank of a node is its location in a list of descending degree order, in which the lowest rank, one, corresponds to the node with the highest degree in the graph. For the case of a tree rooted at a big ISP site, we choose a root node with a low rank, thus ensuring the root is a high degree node with respect to the underlying topology. Then, we either choose the clients as high ranked nodes, or at random, as a control group. Note, that due to the characteristic of the power law distribution, a random selection of a rank has a high probability of choosing a low degree node. The next two tree types have a high ranked root, which corresponds to a multicast session from an edge router. Again, the two types differ by the clients degree distribution, which is either low, or picked at random.

The tree client population is chosen at the range $[50,4000]$ for the 10000 node generated topology, $[50,10000]$ for the 100000 node generated topology, and $[500,50000]$ for the trees cut from real Internet data. For each client population size, 14 instances were generated for each of the four tree types. All of our results are averaged over these instances. The variance of the results was always negligible.

There are two underlying assumptions made in the tree construction. The first, is that the multicast routing protocol delivers a packet from the source to each of the destinations along a shortest path tree. This scenario conforms with current Internet routing. For example, IP packets are forwarded based on the reverse shortest path, and multicast routing protocols such as Source Specific Multicast [19] deliver packets along the shortest path route. In addition, we assume that client distribution in the tree is uniform, as has been shown by [2], [3].

\section{B. Tree Characteristics}

1) Degree-Rank and Size-Rank Power Laws: Our results show that trees cut from a power law topology obey a similar power law. Specifically, we compared the degree-frequency power law found by [4]. Figure 1 shows in log-log scale the degree frequency plot for 


\begin{tabular}{|c|c|c|c|c|}
\hline Name & Type & Parameters & No. of Nodes & Avg. Node degree \\
\hline VS & generated & $a=1 ; p \in 0: 0.05: 0.5$ & 10000 & $1.99-3.98$ \\
IS & generated & $a=2 ; p \in 0: 0.05: 0.5$ & 10000 & $3.99-7.9$ \\
LS & generated & $a=3 ; p \in 0: 0.05: 0.5$ & 10000 & $5.98-12.04$ \\
Big IS & generated & $a=1.5,2 ; p=0.1$ & $50000 ; 100000$ & $3.3,4.4$ \\
BL[1,2] & real data & - & Internet & $3.2^{4}$ \\
LC & real data & - & Internet & $3.2^{5}$ \\
\hline
\end{tabular}

TABLE I

TYPE OF UNDERLYING TOPOLOGIES USED

10000 nodes topology generated with the parameter set $a_{0}=6, a=1, p=0.3, q=0$. The dotted lines here, and in the rest of the linear fit figures, mark the 95\% confidence interval.

Figure 2 shows the same plot for a multicast tree with 500 low degree clients and a root with a high degree. In Table II we summarize the best linear fit parameters in a log-log scale for all trees generated for the topology set $a_{0}=6, a=2, p=0.1, q=0$. It can be seen that the power law holds even for very small trees, e.g., for a tree with 50 multicast clients that has on the average around 200 nodes. The same phenomenon appears in all the trees cut from all topologies, regardless of the way the root and the client nodes were chosen.

These findings conform with the findings of [3], [17] who found a very large frequency of relay nodes in the trees, i.e., nodes with a degree of two. In a power law relationship of frequency and degree, the frequency of two degree nodes is the highest in the tree. Leaf nodes are determined by clients, and are a subset of the clients.

We also found that the distribution of degrees at a specific distance from the root, i.e., in a certain depth ring, also showed a power law distribution of degreerank, but with different slopes.

Given the above findings, it is important to note the following. Cohen et al. [22] showed that the maximal node degree in a graph of $N$ nodes is proportional, for Internet-like topologies, to approximately the square root of the number of nodes. More precisely, $D_{\max } \sim N^{\frac{1}{\alpha-1}}$, where $\alpha$ is the exponent of the degree-frequency power law of the topology. Hence, all resulted degree-frequency graphs of finite sizes exhibit a cut-off at the tail. This holds true for partial views taken from the Internet, with the cut-off being a result of the partiality as well as from the finite size of the Internet itself.

The second power law we found for the trees is of frequency and size of the sub-trees in each tree. Namely, the self similarity holds not only for the degree

\footnotetext{
${ }^{4}$ based on [20]

${ }^{5}$ based on [21]
}

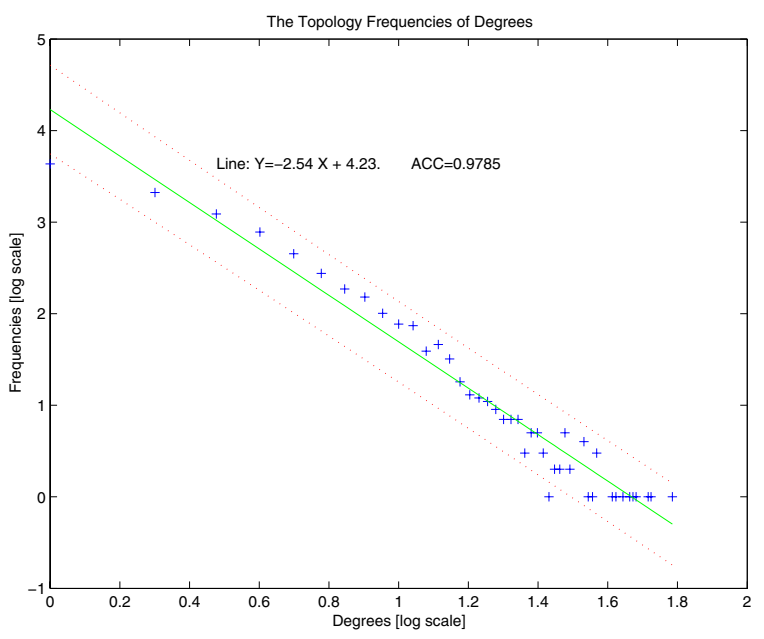

Fig. 1. Frequency of degrees for a 10000 node topology with $a_{0}=6, a=1, p=0.3, q=0$.

distribution in the tree, but also for its inner structure. Figure 3 shows the excellent fit of the complementary cumulative distribution function of the sub-tree sizes of a 2000 Node tree. The tree, with a high degree root, is cut from a 10000 node topology with the parameter set $a_{0}=6, a=2, p=0.1, q=0$. The size distribution differs from the degree distribution in that the big subtrees, although almost similar in size, may differ by one or two nodes, which is negligible compared to their overall size. Thus we give the ccdf graph, which plots the probability that the observed values are greater than the ordinate. It can be seen that the fit to a power law is over $99 \%$. The slope computed for the PDF graph without the tail, resembles the one of the degree distribution.

2) Per Degree Distance Distribution: Cheswick at al. [7] found that the distribution of the number of nodes at a certain distance from a point in the Internet is similar to the Gamma distribution. Our results show that the distribution of distance from the root of nodes of a certain degree seems close to a gamma distribution, although we did not determine its exact nature. Figure 4 


\begin{tabular}{|c|c|c|c|c|}
\hline & $a$ & $p$ & $\mathrm{Y}$ & ACC \\
\hline topology & 2 & 0.1 & $-2.50 X+4.49$ & 0.9721 \\
\hline & \multicolumn{2}{|c|}{ High degree root, low degree clients } & \multicolumn{2}{|c|}{ Root and clients chosen randomly } \\
\hline Receivers & $\mathrm{Y}$ & ACC & $\mathrm{Y}$ & ACC \\
\hline 50 & $-2.76 X+2.25$ & 0.9337 & $-3.27 X+2.68$ & 0.9752 \\
\hline 100 & $-2.64 X+2.42$ & 0.9613 & $-2.96 \mathrm{X}+2.71$ & 0.9611 \\
\hline 300 & $-2.50 X+2.73$ & 0.9730 & $-2.64 X+2.85$ & 0.9717 \\
\hline 500 & $-2.58 X+2.97$ & 0.9732 & $-2.58 X+2.96$ & 0.9654 \\
\hline 750 & $-2.57 X+3.12$ & 0.9825 & $-2.59 X+3.09$ & 0.9609 \\
\hline 1000 & $-2.56 X+3.23$ & 0.9785 & $-2.59 X+3.21$ & 0.9728 \\
\hline 1500 & $-2.64 X+3.45$ & 0.9812 & $-2.56 \mathrm{X}+3.32$ & 0.9741 \\
\hline 2000 & $-2.58 X+3.52$ & 0.9858 & $-2.60 X+3.44$ & 0.9620 \\
\hline 2500 & $-2.65 X+3.66$ & 0.9817 & $-2.63 X+3.57$ & 0.9731 \\
\hline 3000 & $-2.66 X+3.75$ & 0.9851 & $-2.58 X+3.57$ & 0.9670 \\
\hline 4000 & $-2.70 X+3.90$ & 0.9825 & $-2.64 X+3.73$ & 0.9611 \\
\hline
\end{tabular}

TABLE II

LINEAR FIT OF DEGREES AND FREQUENCIES

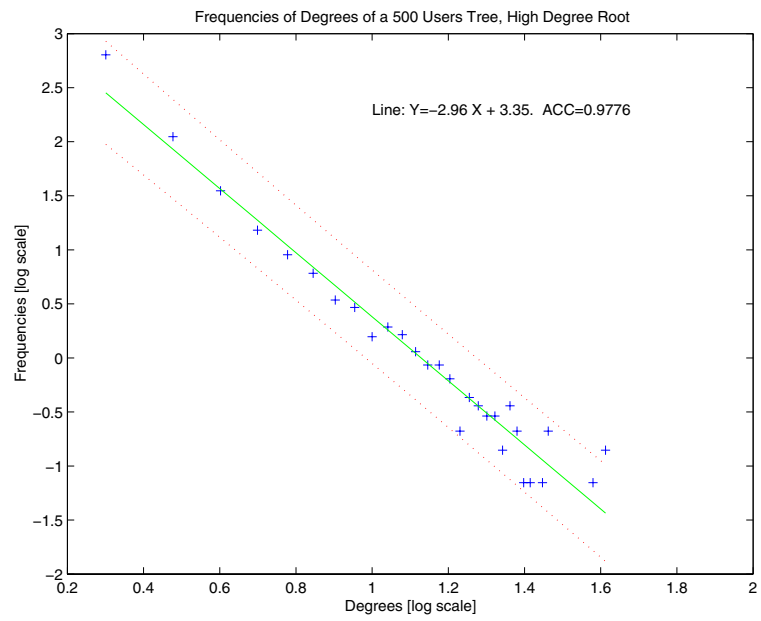

Fig. 2. Tree with 500 low degree clients, high degree root. Cut from topology $a_{0}=6, a=1, p=0.3, q=0$.

shows the distribution of the distance of two to five degree, leaf and high degree nodes, where high degree nodes are nodes with a degree six and higher. In this case the root is a low degree node, and the tree has 1000 low degree clients. As can be seen, the high degree nodes tend to reside much closer to the root than the low degree nodes, and in adjacent rings. In this example, most of them are in the second to forth depth rings around the root.

This phenomenon was even more obvious when the root was a high degree node. We found the following observation with regard to power law generated topologies. The high degree nodes seem to form a 'core'

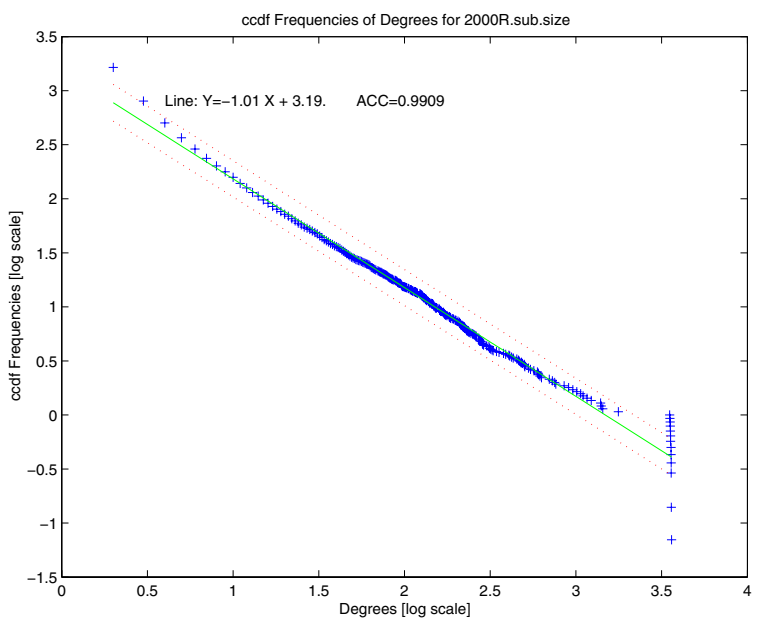

Fig. 3. Sub-tree size CCDF distribution, for a 2000 node tree cut from topology $a_{0}=6, a=2, p=0.1, q=0$.

with a low diameter (around five hops for trees cut from the generated topologies, and seven for trees cut from Internet data) and most of the other nodes in the network are not distanced more than three to five hops away from this core. Subramanian et al. [23] observed a similar phenomenon at the Internet AS topology, although obtained from directed BGP routing tables.

The distribution of client distances from the tree root is given by the leaves distances in Figure 4. Note that the longest path to a client is the tree height. Our results show that the less connected the underlying topology, the taller is the average tree cut from the topology. 


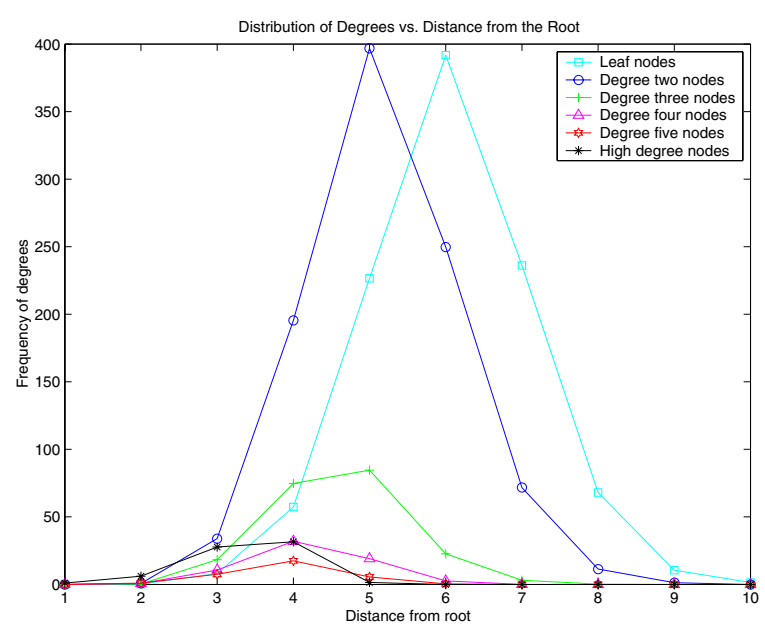

Fig. 4. Distribution of the distance of high degree, two to five degree and leaf nodes in a tree cut from topology $a_{0}=6, a=1, p=$ $0.3, q=0$

3) Empirical Results from Internet Data: We verify the above findings with results obtained from real Internet data. Our results are verified on two different data sets. The first is an Internet partial view at the routers level, obtained from the Lucent Internet Mapping Project [21]. We used this data set as the underlying topology, from which we cut trees in the same manner described in Section II-A. We denote this topology by $L C$.

For the second data set we use the client population of www . bell-labs. com which is a medium size web site. This may represent the potential audience of a multicast of a program with scientific content (such as the livecast of the INFOCOM conference). From this set two lists of clients were obtained, and traceroute was used to determine the paths from the root to the clients. It is important to note, that the first three levels of the tree consist of routers that belong to the site itself, and therefore might be treated as the root point of the tree, although in these graphs they appear separately. We denote this tree as the $B L$ tree.

Figure 6 shows the frequency of degrees for a 10000 node tree cut from the LC topology. The tree, which is an average of 14 instances, exhibits a clear degreefrequency power law with a good fit $^{6}$. The tree was chosen with a high degree root, and low degree leaf nodes. The variance of the instances of each tree was

\footnotetext{
${ }^{6}$ We fit the data for the points above the line $Y=0$ which capture all the degrees that appear on average, at least, once in every tree. To extend the fit below this line we need more trees. If we want to get rid of the noisy tail all together we need to generate, at least, an order of $10^{4}$ trees as our fit predicts that the highest degree points will appear on the average in less than one of every $10^{3}$ trees.
}

negligible, and the same result was obtained for each of the generated trees, with as low as 1000 clients and as high as 50000. Figure 7 shows the frequency of degrees for the BL tree. The linear fit of the log-log ratio is excellent, with a correlation coefficient of 0.9829 .

Figure 5 shows the ccdf of the sub-tree sizes of a tree with 7000 clients cut from the LC data. The root is a high degree node, and the clients are low degree nodes. Note, that every point in the graph is the result of an average of 14 instances therefore the tail was omitted from the fit. The size-rank power law appears in all the trees cut from this data.

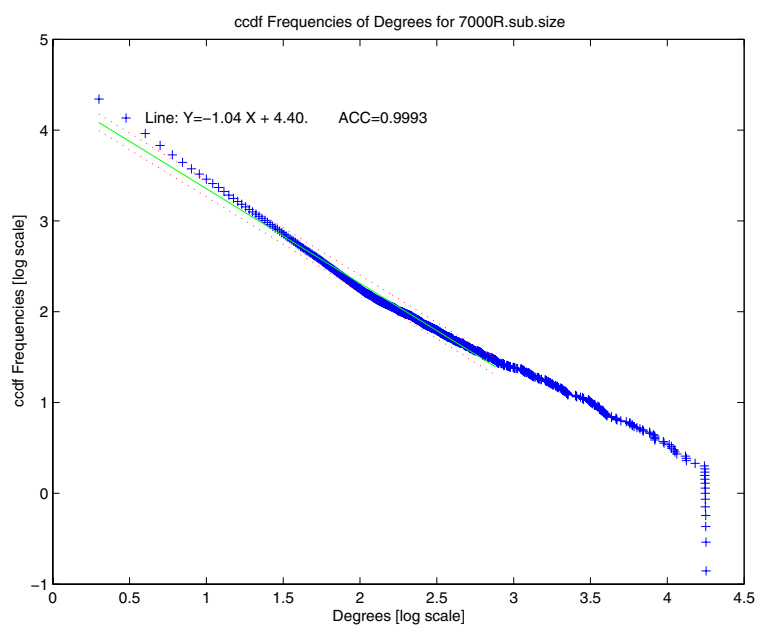

Fig. 5. Size distribution of a 7000 clients tree cut from the LC data

Figure 8 shows the distribution of the distance of two degree, leaf and high degree nodes, for a 15000 client tree, cut from the LC data. The majority (90\%) of the high degree nodes reside within a distance of eight hops from the root, while the clients are distanced up to 18 hops from the root.

\section{Receiver Group Size Estimation Method}

While all of the above observations are interesting and help in our understanding of multicast trees, we were intrigued whether we can use any of this knowledge to evaluate the size of a multicast tree. We compared the degree of the nodes in the tree to their degree in the topology, and focused on the high degree nodes. Interestingly, we found that while some nodes had a tree degree that is significantly smaller than their degree in the underlying network topology, other nodes seemed to have a tree degree close to their network degree. We then compared the frequency of nodes with degree $i$ and above (high degree nodes) to the number of clients in the tree, and found a linear ratio with a correlation 


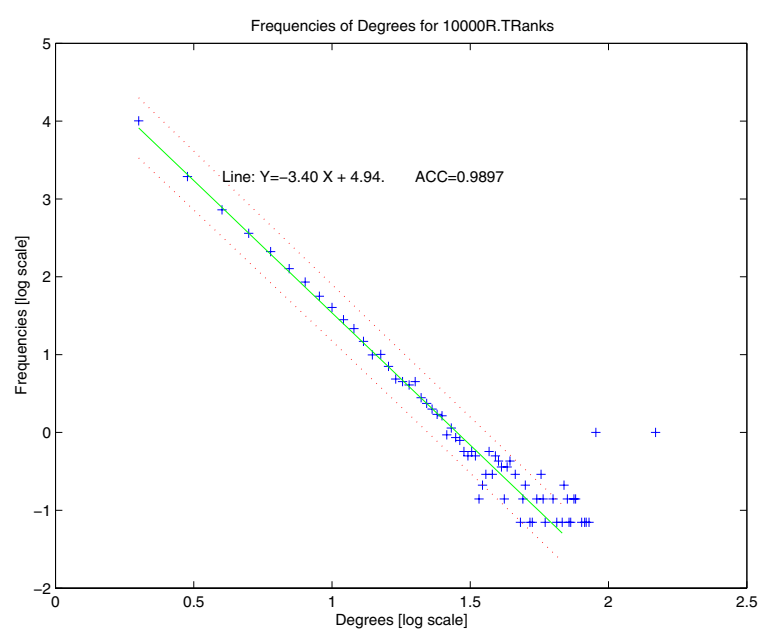

Fig. 6. Frequency of degrees of a 10000 node tree cut from the LC Internet data.

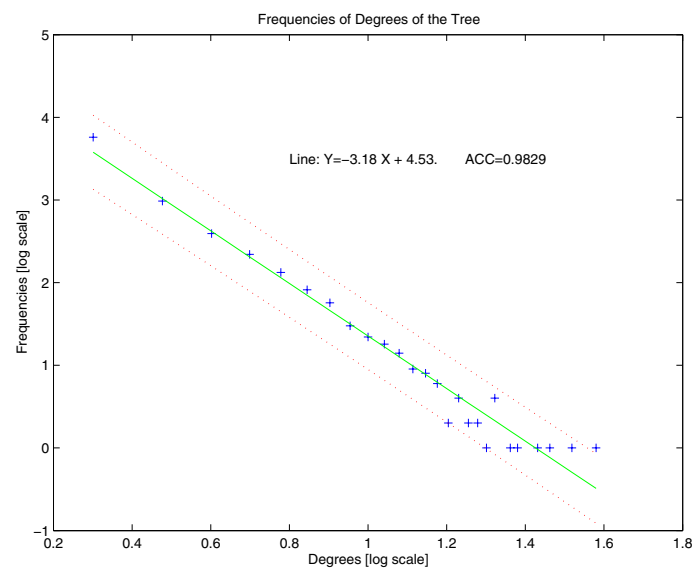

Fig. 7. Frequency of degrees of the BL Internet tree.

coefficient of not less than 0.99 . We term this ratio the $\mathrm{HCN}_{i}$ ratio (hubs-to-client number ratio).

Next, we outline our findings on $\mathrm{HCN}_{i}$ ratio for both simulated trees and trees cut from the real Internet. We proceed by giving a mathematical analysis of our results for power law trees.

\section{A. Empirical Findings}

We have found that an $\mathrm{HCN}_{6}$ ratio of $1: 16$ is a very good predictor for trees cut from the Internet, and most generated topologies. Figure 9 shows the $\mathrm{HCN}_{6}$ ratio in trees cut from a 100000 node topology. The topology parameters are $a_{0}=6, a=1.5, p=0.1, q=0$, and the root node of all trees is a high degree node. The linear ratio is obtained after gathering the information from not more than five depth rings around the root. We plotted the frequency of high degree nodes obtained after

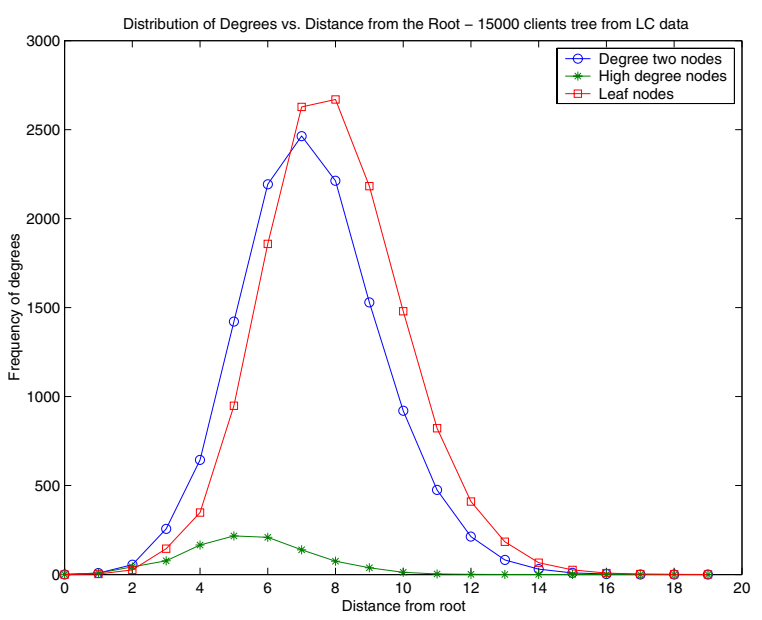

Fig. 8. Distance of two, high degree and leaf nodes from the root of a 15000 client tree cut from the LC Internet data

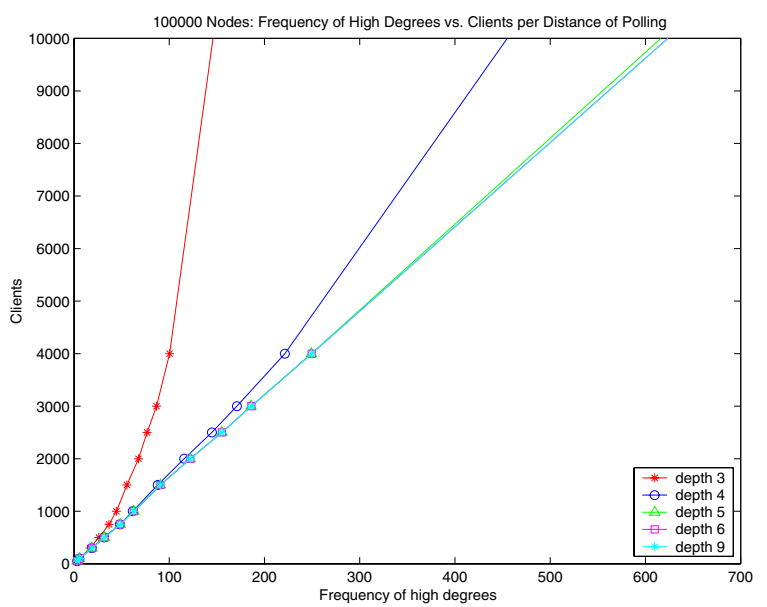

Fig. 9. Clients vs. frequency of high degree nodes. Cut from a 100000 nodes topology with $a_{0}=6, a=1.5, p=0.1, q=0$.

scanning three, four, five, six and nine depth rings around the root. As can be seen from the graph in Figure 9, the entire information was obtained until the sixth depth ring - the following rings did not add any more information. The $\mathrm{HCN}_{6}$ ratio was found to be 16 . Figure 10 shows the excellent fit of the $\mathrm{HCN}_{6}$ ratio with a correlation coefficient of 0.9998 . When we plotted the data for trees cut from this topology with a low degree root, we obtained very similar results. The ratio was again 16, with a correlation coefficient of 0.9996 . However, another depth ring was needed to obtain accurate results, since the root was not as close to the core of high degree nodes as in the previous case.

We verified our results using actual Internet data on the client population of the Bell-Labs web site described in Section II, and on trees cut from the data from 


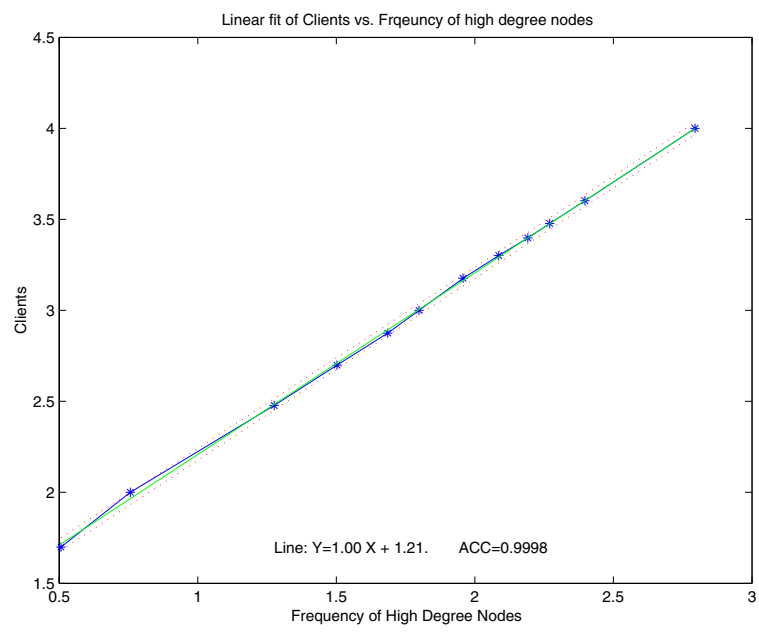

Fig. 10. Linear fit of Clients vs. frequency of high degree nodes. Cut from a 100000 nodes topology with $a_{0}=6, a=1.5, p=0.1, q=0$.

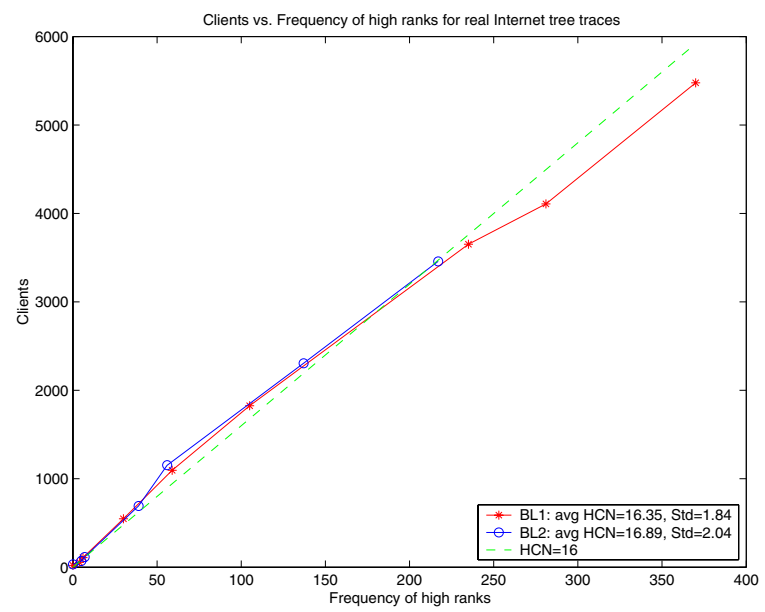

Fig. 11. Clients vs. high degree nodes and the HCN predictor for the $\mathrm{BL}[1,2]$ trees

Cheswick's Lucent Internet mapping project, noted LC, also described there. The Bell-Labs client population data contains two log files. The first, denoted BL1, has 10897 clients and the second, BL2, has 7356. We created subsets of clients by randomly selecting entries from the log files, and cut the corresponding trees for these subsets from the original trees. Figure 11 shows the ratio between the 16 predictor and the actual number of clients in the generated trees. For BL1 the ratio was $1: 16^{0.99}$ with a fit of $99.75 \%$, for BL2 the ratio was $1: 16^{1.04}$ with a fit of $99.72 \%$. For client populations larger than roughly 1500 clients the predictor of 16 gives an excellent estimate - within $9 \%$ of the actual number of clients.

The LC data gives a partial view of the Internet

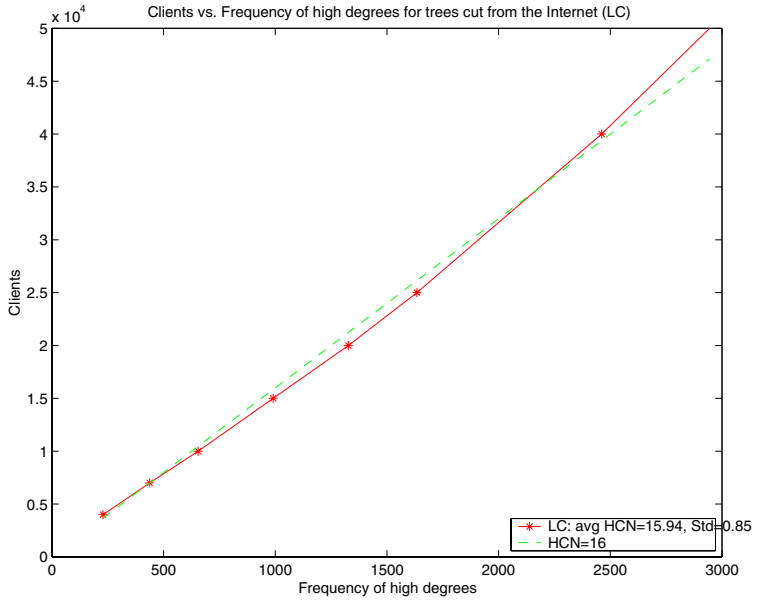

Fig. 12. Clients vs. high degree nodes and the HCN predictor for the trees cut from the LC topology

at the router level with more than 110000 routers. From this topology, we cut trees in the same manner described in Section II. Again, each result is averaged over 14 instances. Figure 12 shows the ratio between the number of clients and high degree nodes, compared with the predicted value from the simulations, 16 . The average value of the ratio is 15.89 , with a standard of deviation of 0.9 . Hence, a 16 predictor for the ratio gives a very good estimation for this data also.

For the generated topologies and the Internet experiments, our results are less definite for very small trees. We found that $\mathrm{HCN}_{6}$ ratio=16 is accurate when client population is at least $0.1 \%$ the size of the underlying topology. Nevertheless, for the Internet, our experiments yielded very good results for group sizes of 1500 clients and more. Note that when the group size is small enough, exact counting of the clients can be done with reasonable cost.

While a predictor of 16 was shown to be a very good predictor for large groups, it becomes less scalable when the group size is extremely large. For example, in the case of a multicast tree with a million clients, the expected number of high degree nodes is 62500. A good solution for this problem is to increase the degree of the sample nodes. For example, in the case of very large groups, counting the number of nodes with degree higher than nine will produce an accurate prediction, with a ratio of 1:48. Note that sampling nodes with a larger degree gives us a coarser estimation. Our experiments show that when we sample nodes of degree ten and above the estimation is accurate only for group sizes of at least $1.5 \%$ the size of the underlying topology. Remember that sampling nodes of degree 6 and above yields a good estimation for trees as small as $0.1 \%$ of 
the network.

\section{B. Analytical derivation of $\mathrm{HCN}_{6}$ ratio}

In this section, we derive the $\mathrm{HCN}_{6}$ ratio for trees in power law topologies. Our experiments have shown that the group of leaf nodes of a tree closely approximates the tree's client population. For simplicity we take the exponent of the underlying topology degree probability instead of the tree's, but these are fairly close.

Given a tree with $N$ nodes, we denote by $L$ the number of leaf nodes and by $\tilde{N}$ the number of non leaf nodes. Let $\tilde{\mathcal{N}}$ be the group of non leaf nodes. The average internal degree is defined by: $r=\frac{\sum_{j \in \tilde{\mathcal{N}}} d_{j}}{\tilde{N}}$ where $d_{j}$ is the degree of node $j$. But by its definition it also holds that $\sum_{j \in \tilde{\mathcal{N}}} d_{j}=2 \tilde{N}+L-1 \approx 2 \tilde{N}+L$, and $\sum_{j \in \tilde{\mathcal{N}}} d_{j}=N+\tilde{N}-1 \approx N+\tilde{N}$. Given all the above we can write

$$
L=N \cdot \frac{r-2}{r-1} .
$$

which holds for any tree.

Given that $p_{i}$ is the probability to find a node with degree $i$ in the tree we can rewrite the above expression for $r$

$$
r=\frac{1}{1-p_{1}} \cdot \sum_{i=2}^{N} i \cdot p_{i} .
$$

and the probability conservation equation

$$
\frac{L}{N}+\sum_{i=2}^{N} p_{i}=1
$$

Substituting (1) in equations (2) and (3), and given that the degree distribution obeys the power law $p_{i}=c \cdot i^{-\alpha}$, we get that:

$$
r=\frac{S_{1}}{S_{2}} \quad ; \quad c=\frac{r}{S_{1} \cdot(r-1)} .
$$

Where $S_{1}=\sum_{i=2}^{N} i^{-(\alpha-1)}$ and $S_{2}=\sum_{i=2}^{N} i^{-\alpha}$. The $\mathrm{HCN}_{6}$ ratio is defined by:

$$
H C N_{6}^{-1}=\frac{\sum_{i=6}^{i=N} p_{i} \cdot N}{L} .
$$

Plugging (1) and (4) in equation (5) yields

$$
H C N_{6}^{-1}=\frac{\left(1-S_{3}\right) \cdot(r-1)}{r-2}-1 .
$$

Where $S_{3}=\sum_{i=2}^{i=5} i^{-\alpha}$.

Figure 13 shows how the $\mathrm{HCN}_{6}$ ratio in equation ( 6) changes with $\alpha$. For $3 \leq \alpha \leq 4$ the $\mathrm{HCN}_{6}$ ratio changes between 14.5 and 19 . Hence, a precise value

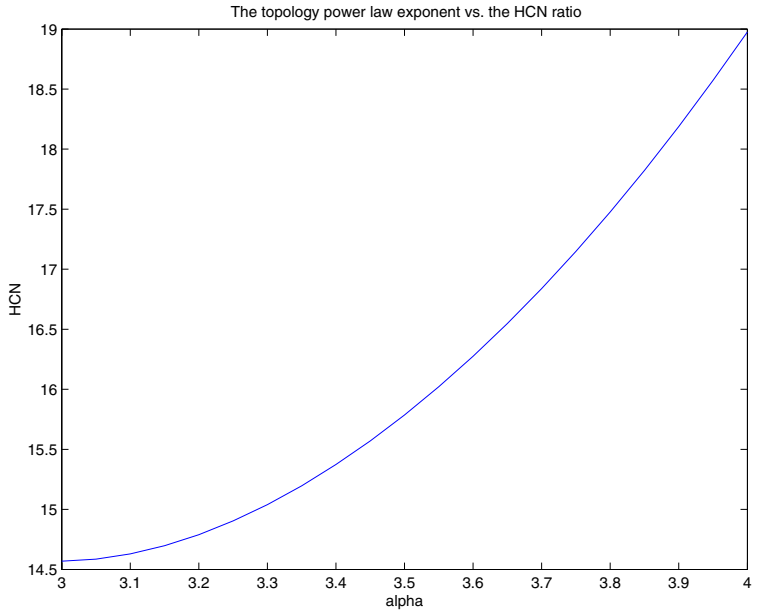

Fig. 13. The change in $\mathrm{HCN}_{6}$ ratio with $\alpha$

for the tree's $\alpha$ will yield an excellent evaluation of the number of leaf nodes in the tree, and hence a good estimation to the client population. Nevertheless, our results show that for the shortest path trees cut from the Internet, as well as from most of our generated topologies, $\mathrm{HCN}_{6}$ ratio $=16$ gives a very good estimation. Understanding the precise correlation between our empiric and analytical results may lead to a deeper understanding of the Internet topology, and is the subject of our next work.

\section{Estimation Algorithms}

\section{A. A Basic Algorithm}

The findings in the previous section give rise to an algorithm for estimating the number of clients in a multicast tree, in which the number of nodes with five or more child nodes is counted. The main idea, given formally in Figure 14, is that the root multicasts a feedback request, Req, along the multicast tree. The request carries the parameter $d$, which indicates the minimal node degree that needs to report back. Such a node, upon receiving the request, replies with a UDP Rep packet sent directly to the root. The root waits for a time long enough to ensure that most replies are accepted. The root then counts the number of different replies it receives, and by multiplying with the appropriate coefficient produces the estimate.

Note that for the Internet, $T_{d_{1}}$, the time the root waits for the replies to arrive, should be quite large. Specifically, $T_{d_{1}}$ needs to be long enough such that the vast majority of slow responses due to round trip and processing delays are not lost. (We assume that $T_{d_{1}}$ of several seconds satisfies these requirements.) 


\begin{tabular}{l} 
Algorithm 1 (Basic) \\
1. Send $\operatorname{Req}(d)$ \\
2. $n \leftarrow 0$ \\
3. Activate $\operatorname{Timer}_{1}\left(T_{d_{1}}\right)$ \\
$\frac{\text { When } \operatorname{Rep} \text { arrives }}{\text { 4. } n++}$ \\
$\frac{\text { When TimeOut }}{\text { 5. return }\left(c_{d} \cdot n\right)}$ \\
\hline
\end{tabular}

Fig. 14. A formal description of the basic algorithm for the root node.

\section{B. Fast Algorithm}

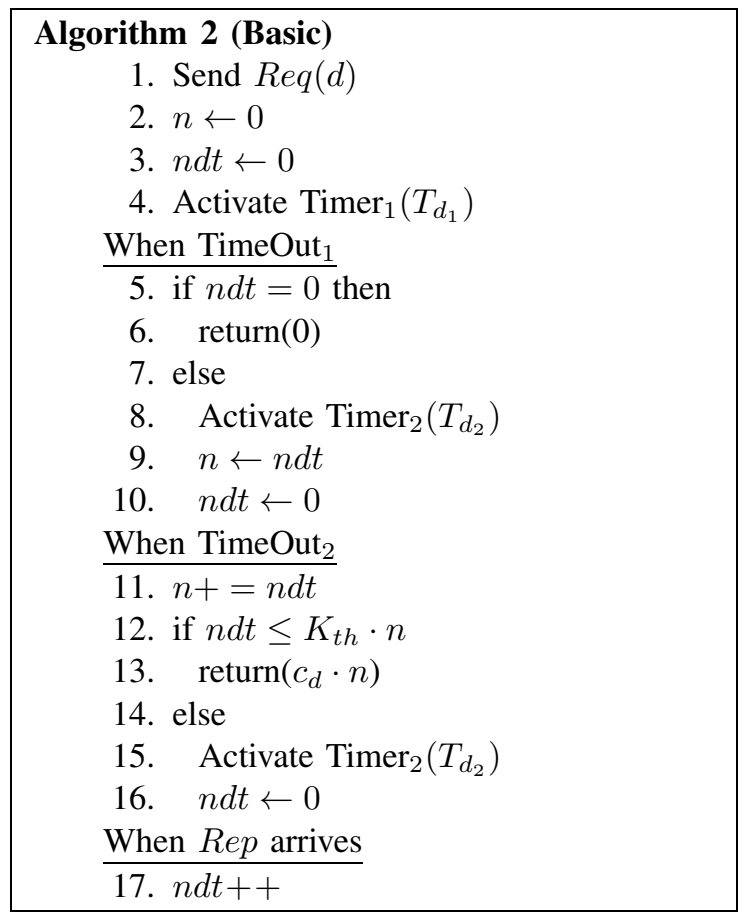

Fig. 15. A formal description of the Fast Algorithm for the root node.

The Fast Algorithm, formally presented in Figure 15 , is motivated by the need to obtain a fast estimation on the client population. We would like to determine the termination rule in a way that guarantees that a significant portion of the Rep messages has already arrived. In the basic algorithm we achieve this by setting a very large timeout. Here, we monitor the Rep message arrival process to achieve this goal.

We start the algorithm with an initial sampling period, $T_{d_{1}}$, whose purpose is to enable responses from the high degree nodes in the $k$-neighborhood of the root to arrive back at the root. If by the end of the initial sampling period the root receives no replies, it assumes the group is either very small or inactive. If the root receives Rep messages, a shorter sampling period termed the iterative sampling period is activated repeatedly until the termination condition is satisfied. The purpose of the iterative sampling period, noted $T_{d_{2}}$, is to enable the algorithm to converge to a good estimate within a short time.

There are several options to determine a termination condition based on the Rep message arrival process. We can choose a threshold and stop when the message arrival rate drops below it. This solution, however, is not immune to network jams, and is very sensitive to the threshold's value. Another option is to stop when the rate keeps dropping for several successive iterative sampling periods. In this case, the algorithm is very sensitive to the length of the iterative sampling period. If it is too short the algorithm might terminate too early with a large estimation error. On the other hand, a long iterative sampling period might cause the algorithm to run longer than necessary.

Thus, we devised a termination rule (see line 12 in Figure 15) that can self-tune according to the arrival process. Under reasonable conditions it will guarantee termination within a preset estimation error. The algorithm terminates when the number of replies received at the root during one of the iterative sampling periods does not improve the estimation by more than $K_{t h}$, where $K_{t h}$ is the estimation error. For example, setting the iterative sampling period to the average two-hop delay and the initial sampling period to $2 T$, causes the algorithm to terminate when the replies gathered from the $T+i$ th depth ring, at the $i$ th iterative sampling period, do not improve the estimation by more than $K_{t h}$. Under reasonable network conditions, about half of the replies from this depth ring reach the root node by the end of the $i$ th iterative sampling period. Thus, the termination condition enables the algorithm to stop when it identifies the end of the adjacent depth rings around the root.

1) Performance Evaluation of the Fast Algorithm: In this section we estimate the delay of the Fast Algorithm and define the average values for $T_{d_{1}}$ and $T_{d_{2}}$. The delay of a packet traversing a single link, $d$, is comprised of two components: $d=\Delta+q$, where $\Delta$ is the fixed minimum link delay and $q$ is a random variable representing the queuing delay, which is exponentially distributed. We would like to derive the distribution of the queuing delay of a packet traveling $h$ links. The density function of the delay, $d_{h}(t)$, is a convolution of the density functions of $q(t-h \Delta), h$ times:

$$
d_{h}(t)=q(t-h \Delta) * q(t-h \Delta) * \cdots q(t-h \Delta) .
$$

Let us define, for simplicity:

$$
\tau=t-h \Delta .
$$


Thus, $d_{h}(\tau)$ is a gamma random variable with parameters $h$ and $\lambda$. Namely:

$$
d_{h}(\tau)=\frac{\lambda^{h} \tau^{h-1} e^{-\lambda \tau}}{(h-1) !}
$$

Where $\lambda^{-1}$ is the average queuing delay. Assuming that all high degree nodes reside within $h$ hops from the root node of the tree, and let the probability of a high degree node to reside at distance $h$ from the root be $p_{h d}(h)$, from Equations (7) and (9) we get that the probability distribution function of the total delay is:

$$
D(\tau)=\sum_{i=0}^{h} D_{h}(\tau) p_{h d}(i)=\sum_{i=0}^{h} \frac{\lambda^{i} \tau^{i} \Gamma(i, i \tau)}{(i \tau)^{i}} p_{h d}(i)
$$

Where $\Gamma(\cdot, \cdot)$ is the incomplete gamma function $[24$, sec. 1.2.11]. Plugging back (8) in (10) we get that the final form of the total delay probability distribution function is:

$$
D(t)=\sum_{i=0}^{h} \frac{\lambda^{i} \Gamma(i, i(t-h \Delta))}{i^{i}} p_{h d}(i) .
$$

The values of $T_{d_{1}}$ and $T_{d_{2}}$ need to be established in a way that will ensure that the majority of the replies are gathered. For example we can select $T_{d_{1}}^{\prime}$ to the value of $t$ that minimizes $D(t)=0.5$, meaning that ensures that on the average we wait for half of the replies to be done waiting at queues.

Alternatively we should chose $T_{d_{1}}$ to be long enough for each node to at least reach the core, preferably its center. Let us define by $r_{c}$ the estimated radius of the core, in which we have established that most high degree nodes reside. Let us define by $r_{e}$ the average distance from an edge node to the core. Then,

$$
T_{d_{1}}=2\left(r_{c}+r_{e}\right)(\Delta+\bar{q})
$$

Thus ensuring that $T_{d_{1}}$ is sufficient for the request to reach the core vicinity and for some of the replies of high degree nodes to arrive back to the root. In the same manner, setting:

$$
T_{d_{2}}=2(\Delta+\bar{q})
$$

yields an iterative sampling period of one hop round trip delay, thus enabling the algorithm to obtain most of the information from the next hop. From our experiments, as described in Section II, we discovered that the values of $r_{c}=7$ and $r_{e}=6$ are sufficient for today's Internet.

In Table III we summarize the simulation results of the Fast Algorithm. We denote by $\tau$ the average one hop delay. The hop delay is either normally distributed (ND) or exponentially distributed (ED). The length of the initial sampling period is $8 \tau$, and the length of the iterative sampling period is $2 \tau$. The results in this table are obtained for trees cut from topology $a_{0}=6, a=$ $1, p=0.3, q=0$, and the Fast Algorithm was executed with an estimation error of $K_{t h}=2 \%$. All the high degree nodes in the generated trees reside within five depth rings from the root. Time units are in $[\tau]$. Note that due to the long tail of the exponential distribution, an iterative sampling period of $2 \tau$ is shown to be too short, since the exponential case represents a bursty network. However, when the delay is normally distributed with variance $\tau$, the algorithm counts all of the high degree nodes in the tree within less than $12 \tau$ time units, which is less than the measured average clients' round trip delay of $16 \tau$ for these trees.

\section{Conclusions}

We presented our findings on the characteristics of shortest path trees cut from power law topologies and the Internet. These findings may improve our understanding of multicast trees and therefore may help theoretical and practical research done in this area. We have shown that the structure of such trees follows power laws of rankdegree and rank-size, and that high degree nodes tend to reside in a low diameter neighborhood.

We found a linear ratio between the number of high degree nodes and the number of multicast tree leaves. We also proved this ratio analytically, and devised the Fast Algorithm that uses this ratio to estimate the tree client population in less than the Internet round trip delay. This algorithm, when used as an initial estimator to polling based counting algorithms such as [11], [13], enables these algorithms to converge much faster, especially for medium and large groups. Note, that these algorithms performance is improved significantly with a tight initial group size estimation. It is also beneficial for transport layer feedback suppression algorithms and control algorithms which need to know the session size such as RTCP [14]. Finally, the Fast Algorithm can be used by network providers in calculating the gain from multicast with metrics such as the one suggested by Chuang and Sirbu [1]. As part of our future work, we intend to include an addition to the Fast Algorithm that enables the root to receive online updates on the changes of the branching characteristics of the trees. These online updates sent by nodes going in or out of the high degree nodes group, enable efficient tracking over time of the multicast group size.

In general, we have found only a few examples where the estimator was off by more than $15 \%$. When the estimator was calibrated to a specific root node the accuracy was a factor of four better. ${ }^{7}$

\footnotetext{
${ }^{7}$ Due to lack of space we omit the details regarding the accuracy and robustness of our predictor.
} 


\begin{tabular}{|l|c|c|c|c|c|c|c|c|}
\hline Clients & 300 & 500 & 750 & 1000 & 1500 & 2000 & 3000 & 4000 \\
\hline ND prediction & 304 & 512 & 736 & 992 & 1472 & 2000 & 2992 & 4000 \\
ND time & 10.0 & 12.0 & 10.0 & 10.0 & 10.0 & 10.0 & 12.0 & 12.0 \\
\hline ED prediction & 256 & 400 & 672 & 960 & 1456 & 1920 & 2736 & 3856 \\
ED time & 12.0 & 12.0 & 18.0 & 14.0 & 20.0 & 18.0 & 16.0 & 20.0 \\
\hline
\end{tabular}

TABLE III

FAST ALGORITHM TIME AND PREDICTION

This work presents a novel way for leveraging topological characteristics of a tree to obtain important knowledge such as its size. A further understanding of the exact ratio between the trees and the underlying topology characteristics is the subject of our future work.

\section{REFERENCES}

[1] J. Chuang and M. Sirbu, "Pricing multicast communication: A cost based approach," in INET'98, Geneva, Switzerland, 1998.

[2] G. Philips, S. Shenker, and H. Tangmunarunkit, "Scaling of multicast trees: Comments on the chuang-sirbu scaling law," in ACM SIGCOMM'99, Cambridge, Massachusetts, USA, 1999.

[3] R. C. Chalmers and K. Almeroth, "Modeling the branching characteristics and efficiency gains in global multicast trees," in IEEE INFOCOM'01, Anchorage, Alaska, 2001.

[4] M. Faloutsos, P. Faloutsos, and C. Faloutsos, "On power-law relationships of the internet topology," in ACM SIGCOMM 1999, Boston, MA, USA, Aug./Sept. 1999.

[5] R. Govindan and H. Tangmunarunki, "Heuristics for internet map discovery," in IEEE Infocom 2000, Tel-Aviv, Israel, Mar. 2000, pp. $1371-1380$.

[6] A. Medina, I. Matta, and J. Byers, "On the origin of power laws in internet topologies," ACM Computer Communications Review, vol. 30, no. 2, pp. 18-28, 2000.

[7] W. Cheswick, J. Nonnenmacher, C. Sahinalp, R. Sinha, and K. Varadhan, "Modeling internet topology," Lucent Technologies, Tech. Rep. Technical Memorandum 113410-991116-18TM, 1999.

[8] H. Schulzrinne, S. Casner, R. Frederick, and V. Jacobson, "RTP: A transport protocol for real-time applications," 1 Jan. 1996, RFC 1899, Internet Engineering Task Force.

[9] S. Floyd, V. Jacobson, S. McCanne, C. Liu, and L. Zhang, "A reliable multicast framework for light-weight sessions and application level framing," in ACM SIGCOMM 1995, New-York, NY, USA, 1995.
[10] D. Rubenstein, J. Kurose, and D. Towsley, "Real-time reliable multicast using proactive forward error correction," in NOSSDAV '98, Berlin, Germany, 1998.

[11] J.-C. Bolot, T. Turletti, and I. Wakeman, "Scalable feedback control for multicast video distribution in the internet," in $A C M$ SIGCOMM 1994, London, UK, Sept. 1994, pp. 58-67.

[12] J. Nonnenmacher and E. W. Biersack, "Optimal multicast feedback," in IEEE INFOCOM '98, San Francisco, CA, USA, Mar. 1998.

[13] T. Friedman and D. Towsley, "Multicast session membership size estimation," in IEEE INFOCOM '99, New York, NY, USA, Mar. 1999.

[14] J. Rosenberg and H. Schulzrinne, "Timer reconsideration for enhanced scalability," in IEEE INFOCOM '98, San Francisco, CA, USA, Mar. 1998.

[15] J. Nonnenmacher and E. W. Biersack, "Scalable feedback for large groups," IEEE/ACM Transactions on Networking, 1999.

[16] A. Alouf, E. Altman, and P. Nain, "Optimal online estimation of the size of a dynamic multicast group," in IEEE INFOCOM '02, New York, NY, USA, 2002.

[17] J. Pansiot and D. Grad, "On routes and multicast trees in the internet," ACM Computer Communications Review, vol. 28, no. 1, pp. 41-50, 1 Jan. 1998.

[18] R. Albert and A.-L. Barabási, "Topology of evolving networks: local events and universality," Physical Review Letters, vol. 85, no. 24, pp. 5234-5237, 11 Dec. 2000.

[19] H. Holbrook and B. Cain, "Source-specific multicast for ip," in Internet Draft,IETF, Feb. 2002.

[20] P. Krishnan, D. Raz, and Y. Shavitt, "The cache location problem," IEEE/ACM Transactions on Networking, vol. 8, no. 5, pp. 568-582, Oct. 2000.

[21] H. Burch and B. Cheswick, "Mapping the internet," IEEE Computer, vol. 32(4), pp. 97-98, 1999.

[22] R. Cohen, K. Erez, D. ben Avraham, and S. Havlin, "Resilience of the internet to random breakdowns," Physical Review Letters, vol. 4626, pp. 85-89, 2000.

[23] L. Subramanian, S. Agarwal, J. Rexford, and R. Katz, "Characterizing the internet hierarchy from multiple vantage points," in IEEE Infocom 2002, New-York, NY, USA, Apr. 2002.

[24] D. E. Knuth, The Art of Computer Programming, 3rd ed. Addison-Wesley, 1997, vol. 1. 